NISSEN, Elke (2004). Expérimentation et présupposés pédagogiques d'un dispositif d'enseignement des langues à distance intégrant le travail de groupe, Études de linguistique appliquée (éla), n¹34, 2/2004, 191-203. (Version soumise)

\title{
Expérimentation et présupposés pédagogiques d'un dispositif d'enseignement des langues à distance intégrant le travail de groupe
}

\begin{abstract}
Résumé. La formation à distance (FAD), et notamment les campus virtuels, se centrent aujourd'hui généralement sur le groupe comme communauté d'apprentissage, dont les bénéfices font l'objet d'un consensus pédagogique. C'est le cas du dispositif de FAD en langues qui fera l'objet de notre étude. Son objectif est l'entraînement des étudiants à une nouvelle certification en langues vivantes dans l'enseignement supérieur (CLES), fondée sur une 'entrée par les tâches'. Notre analyse a pour objet de vérifier le bien-fondé du présupposé pédagogique du dispositif - le travail de groupe - face à une certification portant sur des productions individuelles. Un plan expérimental distingue les étudiants en fonction de leur mode de travail durant une phase d'apprentissage (individuel tutoré $v s$. en groupe tutoré) et compare les acquisitions individuelles dans les deux populations.
\end{abstract}

Au centre de notre observation se trouve la question de l'apport du travail de groupe à distance pour l'acquisition de savoir-faire individuels en langue étrangère. Le présent article expose le contexte dans lequel s'inscrit cette question, et qui relève des choix pédagogiques retenus dans un dispositif universitaire pour l'apprentissage des langues via internet. Un des objectifs du dispositif est d'entraîner les étudiants au CLES, une nouvelle certification en langues dans l'enseignement supérieur qui est en train de se mettre en place. Elle suit la «perspective actionnelle » du Conseil de l'Europe en faisant travailler l'étudiant sur la réalisation de tâches en langue étrangère. Dans le dispositif en question, les étudiants ont le choix de réaliser les tâches individuellement ou de manière collective.

\section{Evaluation et réalisation d'une tâche en langue étrangère}

\subsection{CLES}

L'existence de la certification CLES a été annoncée par un arrêté ministériel du 22 mai 2000 (Lang 2000). Son introduction vise explicitement une modification fondamentale de l'enseignement des langues dans les études supérieures. Mais il poursuit également un autre but : celui d'une meilleure lisibilité des compétences en langues vivantes au niveau européen. Il se réfère ainsi aux dernières publications du Conseil de l'Europe au sujet de l'apprentissage, de l'enseignement et de l'évaluation des langues : le Cadre Européen Commun de Référence pour les langues (Conseil de l'Europe 2001 la première édition est parue en 1996). Une première phase d'expérimentation des modalités du CLES a eu lieu pendant l'année 2001 / 2002. Elle doit être complétée par une deuxième phase d'expérimentation en 2002 / 2003 (reportée finalement à 2003/2004), puis par la mise en place définitive de la certification au niveau national, par les universités qui se portent volontaires (Demichel 2002).

Les trois degrés du CLES correspondent respectivement aux niveaux B1 (degré 1), B2 (degré 2) et C1 (degré 3) des six niveaux des descripteurs de compétences du Conseil de l'Europe. Le CLES tire également du Cadre Européen Commun de Référence pour les langues son « entrée par les 
tâches » (Puren 2001a). A une époque où l'objectif d'apprentissage est de vivre et de travailler avec des étrangers, la visée est sociale. Il s'agit de réaliser une tâche en langue étrangère en contexte social (Puren 2002).

\subsection{La tâche}

Le contexte est dans le cadre du CLES celui des études supérieures : universités, IUFM, instituts techniques, etc. Le type de produits qui sont spécifiques à ce contexte sont par exemple un résumé, une synthèse, un exposé oral ou écrit ou la fiche de lecture d'un ouvrage scientifique. Le CLES évalue la capacité d'un étudiant à réaliser de telles tâches en langue étrangère.

Le dispositif de formation à distance qui servira de cadre à notre expérimentation reprend également le concept de la tâche. La tâche comme unité de l'activité de l'apprenant se trouve au fondement du dispositif observé. Elle représente le point commun entre la future certification des compétences en langues dans l'enseignement supérieur et le dispositif. Cette orientation se profile d'après les textes du Conseil de l'Europe(2001) comme nouvelle approche dans l'enseignement, l'apprentissage et l'évaluation des langues étrangères. Nous définissons la tâche comme authentique (dans le sens où il s'agit d'une activité habituelle dans le cadre des études, $c f$. Conseil de l'Europe 2001 : 121), essentiellement langagière (contrairement à Conseil de l'Europe 2001 : 19, où elle peut également être à composante langagière ou non langagière) et orientée vers un but à atteindre : elle porte sur une production en langue étrangère (parallèlement à Conseil de l'Europe 2001 : 121). Ainsi, dans le dispositif, l'étudiant a la possibilité d'accomplir, seul ou en groupe et avec l'aide d'un tuteur, des productions en langue étrangère qui font sens dans un contexte d'études supérieures. Les savoirfaire mis en œuvre pour les productions relèvent aussi bien des compétences langagières de l'étudiant (la compréhension des documents d'origine ; l'expression orale ou écrite ; le cas échéant l'interaction avec d'autres dans la langue cible) que des savoir-faire plus spécifiquement liés à la nature de la tâche (le respect des règles de cette tâche, e.g. d'un résumé, d'une synthèse) qui sont en jeu et des compétences cognitives de l'individu (trier, extraire, hiérarchiser, réinvestir les idées des documents fournis).

\section{Contexte de la recherche}

\subsection{Travail de groupe}

Le dispositif pédagogique auquel nous venons de faire référence et qui fera l'objet de la partie 3.2. propose aux étudiants deux modes d'accomplissement des tâches, dont l'une est le mode individuel tutoré et l'autre le groupe de pairs tutoré. Nous définissons le groupe comme étant un ensemble d'individus d'un nombre suffisamment restreint pour permettre une interaction effective entre les personnes en vue d'atteindre le but commun qui les rassemble ( $c f$. Anzieu \& Martin 1986 : 161 ; Henri \& Lundgren-Cayrol 2001 : 96). Nous avons choisi le terme travail de groupe au sens d'une activité collective et orientée vers un objectif dans laquelle s'engagent des personnes - ici des apprenants. De sorte que nous ne distinguons pas entre travail collaboratif, qui signifie généralement une activité conjointe et simultanée par les personnes unies, et travail coopératif, qui implique une répartition du travail, suivie par un assemblage des parties (Panitz 1999 : 1-2).

Selon Anzieu \& Martin (1986), le groupe restreint a une dynamique qui lui est propre. Elle est soumise à une tension énergétique, à la fois positive (énergie de production : résolution progressive de la tension en vue d'atteindre un but) et négative (énergie d'entretien: efforts pour améliorer les 
relations interpersonnelles). La communication au sein du groupe est importante non seulement pour l'échange d'informations, mais encore pour le bon fonctionnement du groupe - et donc la minimisation de l'énergie d'entretien. L'énergie « utilisable» du groupe peut ainsi être investie dans la réalisation de tâches collectives.

Le travail en (sous-) groupes, dans l'accomplissement de tâches en langue vivante, est proposé également par le Cadre européen de référence pour les langues:

«e travail en sous-groupes : pour certains apprenants, notamment (mais pas seulement) les
plus lents, le travail en sous-groupe, qui suppose que la compréhension orale ou écrite se fasse
en collaboration, aura vraisemblablement pour effet l'exécution réussie de la tâche, plus que
ne l'aurait eu un travail individuel. En effet, les apprenants se partagent les différentes
opérations de traitement et s'apportent une aide et un feed-back mutuels sur leur
compréhension »(Conseil de l'Europe 1996: chap. 5.3.2.2.; Conseil de l'Europe $2001: 126$ ).

Parallèlement, lorsqu'on parle non de travail collectif mais d'apprentissage collectif, de nombreux courants et théories de l'apprentissage posent l'interaction comme un principe de base de tout apprentissage. C'est le cas par exemple de l'approche socio-constructiviste, de l'approche socioculturelle, de la théorie de l'activité, de l'approche psycho-culturelle, de l'apprentissage situé ou encore de l'approche cognition distribuée (George 2001, Henri \& Lundgren-Cayrol 1998).

\subsection{Travail de groupe à distance}

Si la pratique du travail commun en classe a une longue tradition (e.g. Freinet et Cousinet en France), elle est récente dans la formation à distance (FAD), où elle n'existe que depuis le milieu des années 1990 environ. Son expansion est néanmoins rapide, et liée surtout au développement et à l'amélioration des outils de communication en ligne. De plus en plus nombreux sont aujourd'hui ceux qui pratiquent le CSCW (Computer Supported Collaborative Work), appelé encore en français TCAO (Travail Collaboratif Assisté par Ordinateur) ou plus rarement ACAO (Apprentissage Collaboratif Assisté par Ordinateur). Les campus virtuels favorisent généralement la forme collective du travail, puisque « la synergie du groupe augmente les potentialités pédagogiques de la formation à distance en renforçant le caractère social de l'apprentissage, en valorisant le partage et en favorisant encore plus qu'avant le développement de l'autonomie. (...) S'engager dans des activités d'apprentissage collectives, ne signifie pas s'en remettre aux autres pour apprendre. Chacun doit travailler activement à la construction de ses propres connaissances, mais de manière différente » (Henri \& LundgrenCayrol $1998: 9-10)$.

La «mise à distance » du travail de groupe implique certains changements par rapport à la salle de classe ; elle a ses propres conditions concernant le temps, l'espace, la relation humaine et également l'activité pédagogique. La lecture des textes portant sur ces diverses expériences permet d'établir une liste de conditions pour le bon fonctionnement de la formation, et plus particulièrement du travail de groupe à distance (D'Halluin 2001, Daele 2001, George 2001, Mangenot \& Miguet 2001, Sentini \& al. 2001, Warschauer 2000). Notons cependant que les personnes atteignent plus difficilement un consensus en ligne qu'en face-à-face (Warschauer 1997).

Ces conditions à respecter peuvent être d'ordre technique, telles que les possibilités et le coût d'une connexion à Internet pour une personne ou la facilité d'utilisation des fonctionnalités de la plateforme. Les conditions sont encore organisationnelles et pédagogiques, puisque le niveau de langue de l'apprenant joue un rôle dans son succès dans le travail en groupe, que le tuteur joue un rôle délicat de structuration de la coopération dans lequel il doit savoir doser ses interventions, et que la taille du groupe est, tout comme en face-à-face, déterminante et comprend 4 à 5 personnes dans une configuration optimale. 
Ce qui est également déterminant pour le bon fonctionnement du groupe à distance est le sentiment d'adhésion au groupe (à la 'communauté d'apprentissage') de tous ses membres. Ce sentiment peut être favorisé par un scénario pédagogique adéquat, qui combine différents facteurs tels qu'une communication entre pairs avec feed-back possible, dans la poursuite d'un objectif d'apprentissage explicite, une prise en charge de la responsabilité pour une tâche ou sous-tâche particulière, élaborée selon un échéancier dont les dates sont fixées par les étudiants et un accompagnement pédagogique.

$\mathrm{Si}$ ainsi un certain nombre de points est à respecter pour 'construire le groupe', il reste qu'une fois établi, le groupe peut favoriser un apprentissage efficace, mais aussi prévenir le sentiment d'isolement. Car dans une formation à distance, « dans tous les cas, l'apprenant se trouve confronté à un moment ou à un autre, à une situation d'isolement (réel ou projeté) » (D'Halluin 2001 : 14). Elle paraît inhérente au travail collectif en ligne. Les différents aspects de la distance, physique, temporelle, technologique mais surtout socio-affective sont la cause de ce sentiment d'isolation (Jacquinot 1993). La coopération apparait comme un moyen de diminuer cette impression, voire de la prévenir.

\section{Le dispositif}

\subsection{Question de recherche}

Le dispositif décrit propose aux étudiants de s'entraîner à la réalisation de tâches en langue étrangère en groupe, par le biais des outils de communication proposés (chat, forum de discussion, mél), avec les différentes aides langagières, techniques et méthodologiques, ainsi qu'avec le soutien d'un tuteur. Le bénéfice attendu du travail collectif est de fournir, à travers le partage et la négociation des idées et le retour sur les propositions de chacun, une situation plus propice à l'acquisition que ne l'est la réalisation individuelle d'une tâche. Conformément à la théorie du travail ou encore à la pédagogie de projet de Freinet, il est supposé que l'accomplissement d'une tâche, à plusieurs et en bénéficiant des aides mentionnées, sert à acquérir les savoir-faire nécessaires à la (nouvelle) réalisation d'une telle tâche. La question qui se pose alors est de savoir si l'étudiant apprend en groupe seulement à réaliser une tâche de manière collective ou si les compétences ainsi acquises lui permettent également d'améliorer sa prestation individuelle. Cette question du caractère transférable des compétences acquises en travail de groupe sur une tâche à la réalisation individuelle du même type de tâche en langue cible est significative notamment face à une certification dans laquelle l'évaluation est bien individuelle: le CLES. Notre hypothèse est, globalement, que l'acquisition de savoir-faire langagiers et non langagiers pour la réalisation d'une tâche en langue étrangère est meilleure en mode de travail collectif qu'en mode de travail individuel.

\subsection{Organisation des ressources}

Le dispositif dont il est question ici est un environnement d'apprentissage à distance avec un accompagnement pédagogique. Il a été élaboré par le Service Interuniversitaire de Ressources pour l'Autoformation en Langues (SPIRAL) des universités de Strasbourg. Les langues concernées à l'heure actuelle sont l'allemand et l'anglais. Un des objectifs de ce dispositif universitaire est la familiarisation des étudiants avec le type de tâches demandées dans le certificat de compétences en langues de l'enseignement supérieur (CLES). Le dispositif propose à l'apprenant un ensemble complet 
de ressources et d'outils, ce qui rend le recours à des supports supplémentaires possible mais non obligatoire. Les composantes principales en sont :

- l'autopositionnement - lien vers un test d'autoévaluation téléchargeable et gratuit, en 14 langues, DIALANG (www.dialang.org), qui est développé dans le cadre d'un projet européen et positionne la personne sur les niveaux du Cadre européen de référence (Conseil de l'Europe 1996, 2001) ;

- les outils de communication - chat, forum de discussion, accompagnés de fiches techniques d'installation et d'utilisation, courriel ;

- les ressources authentiques - ressources texte, hypertexte, audio, vidéo, soit sous forme de liens, soit sous forme numérique sur le site, après négociation des droits de publication avec leurs auteurs ou éditeurs. Les ressources peuvent être accompagnées d'exercices d'aide à la compréhension ;

- le cahier de bord - ce cahier est spécifique à chaque étudiant, qui y note ses activités dans le dispositif, les réflexions sur son apprentissage et ses appréciations ;

- l'aide langagière - sélection de liens vers des dictionnaires, grammaires, exercices en ligne ;

- l'aide méthodologique - fiches de conseils et d'application sur des tâches, des compétences (i.e. compréhension écrite, compréhension orale, expression écrite ; une fiche pour chaque tâche proposée, des exercices sur les fiches avec feed-back automatique, etc.) ;

- les tâches de production avec tutorat - travail sur une tâche avec l'aide d'un tuteur (voir 1.2. et 4.1.). Lorsque les étudiants travaillent en groupe, les conditions pour un bon fonctionnement du groupe (indiqués dans 2.2.) sont respectés dans sa constitution et l'accompagnement. Le tuteur a pour rôle de faciliter le travail en groupe, de stimuler le partage d'idées, les interactions et la participation de tous. En même temps, il aide le groupe au fur et à mesure à devenir autonome dans sa gestion et dans sa progression.

\section{Méthodologie}

\subsection{Expérimentation}

Notre programme expérimental implique la comparaison de la qualité des productions individuelles en langue cible après un travail individuel vs. en groupe. La tâche sur laquelle porte l'expérimentation est une synthèse, faite sur un sujet donné et à partir de ressources authentiques, c'est-à-dire non fabriqués à des fins d'apprentissage (articles de presse, articles scientifiques, reportages télévisés, émissions radio, etc.). Il est possible de distinguer différentes étapes successives qui sont nécessaires pour faire une synthèse, et qui sont conseillées aux apprenants dans la réalisation de leur tâche :

- lire, écouter, regarder et comprendre les ressources ;

- identifier les idées et arguments principaux de chaque ressource ;

- recouper les idées principales des différentes ressources entre elles ;

- hiérarchiser ces points forts, leur donner une structure (plan);

- produire un texte cohérent et compréhensible à partir du plan (synthèse) ;

- relire, vérifier et corriger la langue, la cohérence et le contenu de la synthèse. 
Concevoir une synthèse en groupe signifie accomplir conjointement les activités requises lors des différentes étapes, échanger les différentes productions intermédiaires et se mettre d'accord sur les points à retenir ou à changer. Mais au préalable, cela signifie déjà que le groupe soit constitué et enclin à coopérer. Par conséquent, dans un premier temps ont lieu des activités de présentation, de communication ou d'activité commune simple, ainsi que de familiarisation avec l'environnement pédagogique et les outils technologiques. Les activités sont animées par un tuteur qui prend ensuite en charge l'accompagnement du travail de groupe à proprement parler (cf. partie 3.2.). A moins de régler des problèmes urgents, il est important que le tuteur n'intervienne que lorsque le groupe a, dans les différentes étapes intermédiaires, déjà négocié sur les points à retenir. En effet, les apprenants ont tendance à s'adresser en premier lieu au "spécialiste », c'est-à-dire au tuteur (D'Halluin 2001 : 35). Ils interagissent davantage avec lui au lieu de débattre entre eux s'il leur en donne l'occasion (Mangenot \& Miguel 2001 : 261-2). L'interaction entre les étudiants a lieu autant que possible en langue cible. L'échange en langue étrangère favorise l'acquisition de la langue, mais peut également représenter un obstacle dans la négociation sur les différentes étapes d'une tâche à accomplir en commun. Les étudiants peuvent pour cette raison avoir recours à la langue maternelle s'ils n'arrivent pas à dire une chose dans la langue étrangère.

\subsection{Plan d'observation}

Le protocole expérimental porte sur une population étudiante volontaire $(\mathrm{N}=30$ personnes $)$ qui souhaitent améliorer leurs prestations en allemand et qui ont éventuellement déjà pour objectif même si sa mise en place n'est pas encore définitive - de s'entraîner en vue du CLES. Les étudiants sont pour des raisons logistiques obligatoirement inscrits dans une des trois universités strasbourgeoises. Ils peuvent être affiliés à n'importe laquelle des filières proposées dans ces universités. Par conséquent, de même que ce sera probablement le cas dans le deuxième degré du CLES, les ressources retenues sont à tendance scientifique, sans pour autant aborder des champs trop spécifiques (elles porteront $e . g$. sur le thème « Panique créée par les médias ou danger réel ?»).

Comme notre expérimentation a pour but de comparer l'acquisition de savoir-faire en mode de travail de groupe à celle en mode de travail individuel, notre programme expérimental comporte deux populations. La première travaille de manière collective, tandis que la seconde travaille seule. La population «groupe » a à sa disposition des outils de communication (chat, forum de discussion) avec des «salons» privatifs auxquels ne peuvent accéder que les seuls membres de chaque groupe. Ils disposent encore des adresses électroniques de toutes les autres personnes du groupe. Les groupes sont constitués d'environ quatre personnes (voir partie 2.2.). Ils devraient ainsi être suffisamment petits pour permettre une communication efficace à l'intérieur du groupe, tout en étant suffisamment grands pour ne pas multiplier excessivement le nombre des tuteurs.

Le tuteur propose au groupe de suivre les différentes étapes dans l'élaboration d'une synthèse et attire l'attention des étudiants sur l'existence d'une fiche méthodologique avec des conseils pour la synthèse, ainsi que celle des autres aides complémentaires (autres fiches méthodologiques, ateliers méthodologiques, aides langagières, etc.). Il intervient dans la coordination du groupe. En effet, une animation peu directive implique le risque que les étudiants travaillent individuellement, alors qu'une animation directive est facilement trop directive. Pour D'Halluin, le débat reste ouvert s'il faut «favoriser une discussion structurée très tôt ou plutôt commencer par une discussion libre » (D'Halluin 2001: 35). Dans notre cas, l'intervention du tuteur est relativement structurante au départ et s'assouplit au fur et à mesure que les étudiants s'habituent au travail de groupe à distance. 
Les étudiants travaillant de manière individuelle et ceux travaillant en groupe disposent des mêmes aides méthodologiques, langagières et techniques, des mêmes type et fréquence de conseils du tuteur (hormis ceux sur la structuration du groupe), ainsi que du même échéancier. Les ressources servant de base à la synthèse sont identiques, tout comme les thèmes proposés.

Les deux populations réalisent deux synthèses à la suite, afin de bien se familiariser avec les exigences d'une synthèse et de pouvoir réinvestir leurs conclusions sur la meilleure manière de faire lors de la synthèse suivante. La tâche constitue ainsi l'unité de l'activité réalisée par les étudiants. La durée prévue pour chaque synthèse est de quatre semaines. En prévoyant deux semaines de répit entre les deux synthèses, la durée totale de l'expérimentation est de douze semaines.

Préalablement à cette série d'entraînement sur les synthèses, les étudiants des deux populations rédigent une synthèse de manière individuelle. Pour ce pré-test, ils disposent d'indications précises sur la nature, la forme et la longueur attendue de leur rédaction. Le nombre de documents proposés, les conditions de passation, la durée de l'épreuve, etc. sont conformes aux exigences du degré 2 du CLES (voir Lang 2000, annexe 2). La rédaction sert à évaluer leur capacité à réaliser la tâche en langue étrangère avant de s'être entraîné dans le cadre du dispositif en ligne. Puis, suite à la série de deux synthèses, chaque étudiant rédige de nouveau de façon individuelle une autre synthèse. Le résultat à ce post-test nous permet de mesurer leur compétence à réaliser la tâche en langue cible à la fin de l'expérimentation. La comparaison entre les prestations au pré-test et celles au post-test, en distinguant les populations «en groupe » et «individuelle», se fera au moyen du test statistique de l'analyse de la covariance. Elle nous permettra de tirer des conclusions sur l'apport du travail de groupe dans l'acquisition de savoir-faire individuels pour la réalisation d'une tâche - la synthèse - en langue étrangère.

\subsection{Critères d'évaluation des productions individuelles}

Les capacités mises en œuvre dans la rédaction d'une synthèse - et avec elles les critères d'évaluation - ne sont pas seulement d'ordre langagier (voir 1.2.). L'approche communicative a vu apparaître des critères appelés 'pragmatiques' portant sur l'efficacité de la communication - puisque c'est la communication qui était au centre de l'enseignement / apprentissage durant cette période (Puren 2001a: 19). Dans une approche comme la perspective actionnelle du Conseil de l'Europe (1996 / 2001), où la langue prend une fonction d'outil d'action, l'efficacité de l'action, et donc le critère pragmatique, garde toute son importance. L'expérimentation dans le cadre de CLES n'a pas encore apporté de réponse définitive aux critères d'évaluation d'une prestation du type tâche. Une proposition pour une catégorisation des critères d'évaluation des tâches faite par Puren (Puren 2001b) - et qui a servi de base pour les réflexions dans le cadre de l'expérimentation CLES - comporte également les critères pragmatiques, définis comme «réalisation de la tâche, adaptation aux contraintes, adéquation du produit à la demande, efficacité du résultat». Outre les critères langagiers et pragmatiques, il propose des critères cognitifs, relatifs aux opérations intellectuelles mises en œuvre. Cette aptitude intellectuelle permet l'exploitation et la réutilisation des ressources ainsi que, globalement, la réalisation des tâches. Nous ne retenons pas le dernier type d'exigences retenu, c'est-àdire les critères disciplinaires, relatifs au domaine de spécialité et à ses règles. En effet, la spécialisation disciplinaire n'est pas en vigueur dans le dispositif en question.

Ainsi, les critères retenus pour notre évaluation sont premièrement les critères langagiers :

- l'expression écrite et le style dans la rédaction finale : reformulation des idées provenant des documents authentiques, variation du vocabulaire, maitrise grammaticale, correction et 
richesse syntaxique ainsi qu'emploi des mots de liaison, qualité de l'orthographe (la compréhension orale et écrite n'est pas évaluée directement, mais est un présupposé pour pouvoir reconnaître les informations importantes).

Deuxièmement, les critères d'évaluation de la tâche sont pragmatiques :

- cohérence de la synthèse (identification de la problématique, organisation du discours, présentation claire et structurée de la problématique), agencement des idées (et des parties), clarté et adéquation de l'introduction et de la conclusion à la partie principale de la rédaction.

Les critères sont, troisièmement, d'ordre cognitif. Les compétences cognitives sont importantes dans le processus d'élaboration de la tâche, mais sont étroitement liées aux compétences langagières et pragmatiques. Elles sont par conséquent difficiles à évaluer séparément dans une analyse du résultat final, c'est-à-dire de la production écrite. Nous n'avons ainsi retenu comme indice de la performance cognitive que :

- l'identification des idées pertinentes dans les ressources fournies et leur intégration dans la rédaction.

Autrement dit, le travail sur la tâche fait appel aussi bien à des savoir-faire langagiers qu'à des savoir-faire pragmatiques et cognitifs qui sont imputables au type de tâche, mais non au fait qu'elle soit réalisée en langue étrangère. Les compétences cognitives requises sont les mêmes pour une synthèse en langue maternelle. Les compétences pragmatiques, quant à elles, ne peuvent être mises en œuvre qu'au moyen d'une certaine maitrise de la langue - or, s'exprimer de manière cohérente et claire dans une rédaction demande également des compétences particulières, non langagières. Distinguer différents types de critères signifie distinguer également deux niveaux d'apprentissage : celui de la langue et celui du savoir-faire propre à la tâche.

Se poser la question des apports du travail de groupe pour la réalisation de tâches en langue étrangère implique un questionnement aussi bien sur les apports langagiers que sur les apports pragmatiques et cognitifs. Lorsqu'ils écoutent, lisent et rédigent en langue étrangère tout en s'entraînant sur un certain type de tâche, les apprenants améliorent-ils autant leurs compétences langagières que leurs compétences pragmatiques et cognitives ? Afin de répondre à cette question, la comparaison prévue entre le pré-test et le post-test distingue les critères langagiers, pragmatiques et cognitifs. L'observation comporte par conséquent deux variables dépendantes : le mode de travail (en groupe $v s$. individuel) et la nature des savoir-faire (langagiers, pragmatiques ou cognitifs).

\section{Résultats et analyse de l'expérimentation}

Les productions des étudiants ont été évalués par deux correcteurs distincts, qui ont attribué des scores séparément pour les trois aspects mentionnés ci-dessus : la qualité langagière, pragmatique et cognitive des rédactions. Les performances des étudiants au pré- et au post-test ont été comparées à l'aide des tests statistiques de la variance ou bien de la covariance, une fois qu'il a été vérifié que les conditions d'applicabilité des tests sont remplies. Il apparaît, dans le cadre de cette étude, menée dans le contexte plus haut, que la supériorité de l'apprentissage en groupe tutoré par rapport à l'apprentissage individuel tutoré ne peut pas être affirmée.

Un trop grand écart de départ entre les populations ne nous a pas permis l'évolution de l'aspect cognitif. En ce qui concerne l'aspect langagier, aucun progrès entre le pré- et le post-test n'a pu être constatée pour les deux populations actives. Au niveau pragmatique, par contre, un 
apprentissage peut être observé. Or, la population qui améliore le plus sa performance n'est pas celle du groupe tutoré, comme nous le supposions, mais la population individuelle.

Une évaluation des productions que les étudiants ont réalisées pendant la phase d'apprentissage a complété notre programme expérimental. Elle est basée sur la même grille d'évaluation que celle des deux tests en amont et en aval de la formation. Elle suggère que le groupe est un meilleur moyen pour produire que pour apprendre. En effet, lorsqu'une différence entre les populations existe, elle se dessine en faveur du groupe, à l'inverse des situations de test - individuelles - observées. Cela est le cas pour l'aspect ...\#

Au centre de notre recherche se trouve la question de savoir si les compétences acquises en groupe sont transférables à la réalisation individuelle d'une tâche, et plus particulièrement d'une synthèse, en langue étrangère. Les observations faites dans le cadre de notre recherche seraient à vérifier par d'autres études, en variant le public, les tâches et le scénario pédagogique retenus. Elles suggèrent néanmoins que l'adéquation pédagogique entre la proposition d'un apprentissage conjoint entre pairs avec un tuteur et une évaluation individuelle, faisant abstraction de la composante sociale, est à mettre en question. Elles nous font également penser que, plutôt que de considérer le groupe $a$ priori comme la meilleure solution possible pour une formation à distance - en langues ou non -, il sera utile de s'interroger sur ses apports, par rapport à cet autre mode faisant aussi appel à l'interaction sociale en ligne qui est l'apprentissage individuel tutoré. Ce mode n'est en effet généralement pas envisagé comme une réponse aux questions que la recherche du meilleur scénario possible dans une FAD pose.

(\#) Notre étude, en donnant un statut central à la perspective actionnelle européenne, vérifie expérimentalement le principe de la tâche dans un contexte particulier : celui de la formation à distance avec une modalité de travail de groupe. La réflexion sur une participation au CLES via internet a fait partie intégrante de sa première phase expérimentale. Le dispositif décrit, et avec lui notre recherche, permet de pousser plus loin cette réflexion et de tester la faisabilité d'un travail sur la tâche à distance, idéalement même d'en déterminer les modalités.

\section{Bibliographie}

Anzieu, Didier, Martin, Jacques-Yves (1986). La Dynamique des groupes restreints, Paris, PUF, 8e édition revue et corrigée (C 1968).

Conseil de l'Europe (1996), Conseil de l'Europe, Les Langues vivantes : apprendre, enseigner, évaluer. Un Cadre européen commun de référence, Strasbourg, 1996.

Conseil de l'Europe (2001), Conseil de la Coopération culturelle, Comité de l'éducation, Division des langues vivantes Strasbourg, Cadre européen commun de référence pour les langues apprendre, enseigner, évaluer, Paris, Conseil de l'Europe / les Editions Didier, 2001.

Daele, Amaury, Khamidoullina, Izida, (2001). Un campus virtuel soutenant la collaboration, in De Vries \& al., pp. 362-4.

De Vries, E., Pernin, J.-Ph., Peyrin, J-P. (éds.) (2001). Hypermédias et Apprentissages, Actes du 5 e colloque, INRP, EPI.

Demichel, Francine (directrice de l'enseignement supérieur), (2002). Lettre circulaire du 29 avril 2002 du ministère de l'éducation nationale.

D'Halluin, Chantal (coord.) (2001). Usages d'un environnement médiatisé pour l'apprentissage coopératif, Lille, CUEEP. 
George, Sébastien (2001). Apprentissage collectif à distance, SPLACH: un environnement informatique support d'une pédagogie de projet, thèse de doctorat, Université du Maine.

Henri, France, Lundgren-Cayrol, Karin (1998). Apprentissage collectif et nouvelles technologies, rapport, centre de recherche LICEF, bureau des technologies d'apprentissage, http://oltbta.hrdc-drhc.gc.ca/projects/completed/NPLT/69018final_f.pdf (dernière consultation 29/05/02).

Henri, France, Lundgren-Cayrol, Karin (2001). Apprentissage collaboratif à distance. Pour comprendre et concevoir les environnements d'apprentissage virtuels, Québec, Presses de l'Université du Québec.

Jacquinot, Geneviève (1993). Apprivoiser la distance et supprimer l'absence ? ou les défis de la formation à distance, Revue Française de Pédagogie 102, Paris, pp. 55-67.

Lang, Jack, (2000). Certificat de compétences en langues de l'enseignement supérieur, arrêté ministériel du 22 mai 2000, Bulletin Officiel du ministère de l'Education Nationale et du ministère de la Recherche, $\mathrm{n}^{\circ} 25$ du 29 juin 2000, http://www.education.gouv.fr/bo/2000/25/sup.htm (dernière consultation 16/11/03).

Mangenot, François, Miguet, Mathilde, (2001). Suivi par internet d'un cours de maîtrise à distance : entre individualisation et mutualisation, in De Vries \& al., pp. 259-266.

Panitz, Theodore (1999). Collaborative Versus Cooperative Learning: A comparison of the two concepts which will help us understand the underlying nature of interactive learning, The ERIC Clearninghouse on Assessment and Evaluation, University of Maryland, http://ericae.net/k12assess/colcoo.htm (dernière consultation 16/11/03).

Puren, Christian (2000). Champ sémantique de 'tâche'. Document remis au séminaire «Didactique des langues et technologies éducatives », 2001/2002, UTC, Compiègne.

Puren, Christian (2001a). La problématique de l'évaluation en didactique scolaire des langues, Les Langues modernes $n^{\circ}$ 2/2001 (avril - mai - juin), Dossier : évaluation et certification en langues, Paris, APV, pp. 12-29.

Puren, Christian (2001b). Certificat de compétences en langues de l'enseignement supérieur (CLES). Critères d'évaluation des tâches (projet), document de travail remis lors d'une réunion des concepteurs expérimentaux de CLES le 8 juin 2001 à Paris Dauphine, 1 page.

Puren, Christian (2002, à paraître). Chronique d'une évolution méthodologie annoncée: la 'perspective actionnelle', TESOL France.

Senteni, Alain, Aubé, Michel, Defresne, Aude (2001). Un modèle de support au travail collaboratif dans un centre virtuel d'apprentissage, in De Vries \& al., pp. 225-239.

Warschauer, Mark (1997). Computer-Mediated Collaborative Learning: Theory and Practice, http://www.gse.uci.edu/markw/cmcl.html (dernière consultation 16/11/03).

Warschauer, Mark (2000). On-line learning in second language classrooms : An ethnograhic study, in Warschauer, M., Kern, R., Network-based Language Teaching: Concepts and Practice, Cambridge University Press, pp. 41-58. 